\title{
Efficient algorithms and models for mechanical and structural design optimization
}

\author{
M. M. Kasem ${ }^{1}$ and K. Y. Maalawi ${ }^{2}$ \\ ${ }_{1}^{1}$ Aerospace Engineering Department, Cairo University, Giza 12613, Egypt \\ Phone: +201061154412 \\ 2 Department of Mechanical Engineering, National Research Centre, Dokki, P.O. 12622, Cairo, Egypt
}

ABSTRACT - In the present work, different algorithms and penalty methods for design optimization of mechanical elements and structures are applied. Seven robust optimization techniques and seven penalty methods are thoroughly investigated and implemented in MATLAB codes. In addition, different optimization models are compared using two benchmark problems, namely, the minimal cost design of a welded beam structure and the optimal buckling design of a functionally graded material column. A performance measure factor is defined to determine the best technique among the implemented optimization algorithms. The results are arranged and nested to make it easy for the reader to figure out each technique characteristics, and hence choose the suitable one for a specific design problem and/or application. Comprehensive computer experimentations were performed, and the best optimization techniques and models have been thoroughly demonstrated. The attained optimal solutions show that, in general, the hybrid algorithms worked better than the stand-alone ones and the sequential quadratic programming (SQP) with global search indicates a superior performance than other techniques. Finally, based on the present study, the adaptive and dynamic penalties need further investigation to become more consistent with the implemented optimization algorithms.
ARTICLE HISTORY

Received: $21^{\text {st }}$ Mar 2020

Revised: $30^{\text {th }}$ Jan 2021

Accepted: $25^{\text {th }}$ Mar 2021

\section{KEYWORDS}

Optimization and penalty

methods;

hybrid algorithms;

structural optimization

models;

mechanical design;

functionally graded

materials.

\section{INTRODUCTION}

Optimization has become a cornerstone in numerous engineering fields such as aerospace, mechanical, civil and ocean engineering [1]. Scientists have been using several optimization techniques to acquire the best combination of some design variables for certain preferences. With the vast number of optimization algorithms available worldwide, it is not easy for a designer to determine an appropriate algorithm to solve certain optimization model. The present work investigates the effect of using different optimization models and algorithms on the optimal design of a specific engineeringproblem.

The standard form of the optimization model considered in the present work is cast in the following:

Find the design variable vector $\vec{x}=\left(x_{1}, x_{2}, \ldots \ldots, x_{N}\right)^{T}$, which:

$$
\begin{array}{lll}
\operatorname{Minimize} f(\vec{x}) & & \\
\text { Subject to } & g_{j}(\vec{x}) \leq 0, & j=1,2, \ldots \ldots, J \\
& h_{k}(\vec{x})=0, & k=1,2, \ldots \ldots, K \\
& x_{i}{ }^{L} \leq x_{i} \leq x_{i}{ }^{U}, & i=1,2, \ldots \ldots, N
\end{array}
$$

where $f(\vec{x})$ is the objective function, $g_{j}(\vec{x})$ are the inequality constraints, and $h_{k}(\vec{x})$ are the equality constraints. $x_{i}{ }^{L}$ and $x_{i}{ }^{U}$ are the lower and upper limiting values imposed on design variables (side constraints). Eq. (1) represents a general constrained nonlinear optimization problem [1].

Several research works can be found in literature comparing various optimization methods. Venter [2] introduced a general review of different optimization algorithms and their classifications. He classified them as local and global; constrained and unconstrained; and gradient and non-gradient methods. This kind of revision gives the reader a general overview of the optimization methods available and their characteristics. Yeniay[3] compared the performance of three optimization methods for constrainednonlinear problems. He compared the sequential quadratic programming (SQP), the generalized reduced gradient (GRG) and the genetic algorithm (GA) using fifteen benchmark problems. The genetic algorithm was found to be the best algorithm among other methods. GA was also compared with the Particle Swarm Optimization (PSO) for trajectory optimization [4], where it was found that GA has less execution time than the PSO. However, in some cases, the PSO can be more accurate than the GA such as in bi-level linear programming problems outlined in [5]. Fan and Zahara [6] compared four optimization algorithms: the Nelder-Mead simplex algorithm (NM), the Particle Swarm Optimization (PSO), the Guaranteed Convergence Particle Swarm Optimization (GCPSO) and the hybrid Nelder-Mead Particle swarm algorithm (NM-PSO) using 20 benchmark problems. They found the (NM-PSO) is the best in robustness, accuracy and number of function evaluations. Such hybrid form has shown to be supperior than 
using the (PSO) alone. Ren [7] improved the well-known simple genetic algorithm (SGA) and renamed it the improved genetic algorithm (IGA), which was combined with the Nelder-Mead algorithm (NM- IGA) resulting in a much better computational efficiency.

In general, an optimization process is twofold; first, it improves the performance of the objective function, and second, it obtains the best function value subject to some constraints. These goals cannot be achieved without using the proper optimization technique in addition to proper optimization model. The main focus of the present work is to study the effect of changing the optimization algorithm as well as the optimization model on the attained optimal solutions of a specific design problem. Seven different optimization methods are considered, ranging from simple to complex, single to hybrid, and local toglobal. These methods are the (NM), (PSO), (GA), (SQP), hybrid (NM-PSO), (NM-GA), and (PSO-GA). All these techniques are implemented in MATLAB codes and experimented using carefully selected benchmark problems.

Seven penalty functions for constrained optimization are examined and tested aiming at investigating the effect of these penalty methods on the optimization performance and determining the characteristics of each algorithm in comparison with others. This will help select the best algorithm among them that is expected to be the most efficient and robust according to the present applications.

\section{METHODS AND MATERIALS}

The most commonly applied optimization algorithms are based on random search such as NM, PSO, and GA, while others are gradient-based, such as the SQP. In general, they can be classified into two categories: stand-alone algorithms and hybrid algorithms. A brief discussion of the two types is given in the following sections.

\section{A-Standalone (Single) Algorithms}

The Nelder-Mead method (NM) [8] is one of the simplest optimization methods. It is based on comparing the values of the objective function at $N+1$ points ( $N$ represents the number of design variables) and moving gradually towards the minimum point using four operations; reflection, expansion, contraction, and shrinking. It is an unconstrained optimization in which any constraint should be implemented as a part of the objective function. The NM is a local, nongradient method, as it is affected by local minima.

The Particle Swarm Optimization (PSO) simulates the behavior of a swarm or colony of birds in which when one member of the swarm finds a preferred point, the other particles move with certain speed towards this point. It is a simple and powerful method like the (NM), and it is classified as a global optimization technique. PSO was first introduced by Eberhart et al. [9] in 1995 to present a relatively simple model that can solve a wide range of problems. Bai [10] presented some improvements to the original (PSO) which are considered in the present work.

The GA is originally designed for the study of adaptive systems rather than optimization, then it becomes one of the popular methods in design optimization. It is classified as a global, non-gradient optimization method that is based on the process of natural selection. The GA was first proposed by Holland [11] in 1975s. From this time, many developments have been made to make the GA faster and more efficient. Guo and Yang [12] introduced a modified version of the simple or conventional genetic algorithm that is considered in this study.

The Sequential Quadratic Programming (SQP) is based on the minimization of a quadratic model using a quadratic programming sub-routine. It was first developed by Wilson [13] in 1963 in his $\mathrm{PhD}$. After then, many evolutions have been made to the method to make it more powerful and convenient. According to Venter [2], it is a gradient-local method that is widely used in many engineering applications, however there is a global version available in MATLAB toolbox. More details about SQP can be found in [14]. Seeking simplicity in the current work, the MATLAB built-in SQP function "fmincon" is implemented. Table 1 summarizes the main characteristics of the previous four optimization techniques.

\section{B - Hybrid Algorithms}

One way to develop a strong, robust optimization method is to combine two different optimization algorithms. Examples such as the (NM-PSO), (NM-GA), and (PSO-GA) were proposed by researchers in the field. Such hybrid methods, for most cases, combine the advantages of their individuals and overcome their disadvantages. For instance, the NM method is a straightforward and computationally efficient method of optimization. However, it is highly affected by local minima [15]. On the other hand, the Genetic Algorithm is based on global search without using local information. The combination of these two methods is expected to combine the advantages of both algorithms. Ren [7] and Rahami et al.[15] provide detailed description of the hybrid (NM-GA) algorithm that is expected to obtain more accurate results than any of its standalone techniques. The improved version of GA developed by Ren [7] will be applied herein. Al-Garni and Kassem [4] proposed a hybrid (PSO-GA) that combines the best of the PSO and GA. According to them PSO-GA approved superior execution time and performance. Fan and Zahara [6] developed a hybrid (NM-PSO) optimization algorithm and applied it successfully in finding the optimum solution of certain constrained engineering design problems. 
Table 1. Comparison among the NM, PSO, GA, and the SQP

\begin{tabular}{|c|c|c|c|c|}
\hline Method & $G A$ & PSO & $N M$ & $S Q P$ \\
\hline $\begin{array}{c}\text { Year of } \\
\text { development }\end{array}$ & 1975 & 1995 & 1975 & 1963 \\
\hline Approach based on: & $\begin{array}{l}\text { The process of } \\
\text { natural } \\
\text { selection }\end{array}$ & $\begin{array}{l}\text { Swarm of birds } \\
\text { behaviour }\end{array}$ & $\begin{array}{l}\text { Four operations; reflection, } \\
\text { expansion, contraction, and } \\
\text { shrinking }\end{array}$ & $\begin{array}{l}\text { The minimization of a } \\
\text { quadratic model }\end{array}$ \\
\hline Search technique & Random & Random & Random & Gradient \\
\hline Optimal Solution & Global & Global & Local & Local/Global \\
\hline
\end{tabular}

\section{C- Constraints Handling Techniques - The Penalty Function Method}

In constrained optimization, care should be taken to the representation of design constraints. There are several methods found in literature for constraint representation. Seven methods are investigated and compared. Six optimization techniques are used in the present comparison: the NM, PSO, GA, (NM-PSO), (NM-GA), and (PSO-GA) to investigate the effect of using each constraint handling technique on each of them.

In non-gradient search methods, the most popular technique to handle the design constraints is the penalty method, in which the constraint problem is transformed into unconstraint one, in the form [16]:

$$
\begin{gathered}
\text { Minimize } \quad F(\vec{x})=f(\vec{x})+P(\vec{x})=f(\vec{x})+(\text { Penalty parameter }) E(\vec{x}) \\
\text { where } \quad E(\vec{x})=\left(\sum_{j=1}^{J} g_{j}{ }^{\beta}(\vec{x})+\sum_{k=1}^{K} h_{k}{ }^{\beta}(\vec{x})\right)
\end{gathered}
$$

$F(\vec{x})$ is the equivalent cost function, $P(\vec{x})$ is the penalty function, and $\beta$ is a penalty parameter. The efficiency of the penalty function $P(\vec{x})$ depends on the proper selection of the penalty parameters such as the exponent $\beta . E(\vec{x})$ in the $2^{\text {nd }}$ equation represents the summations of the constraint functions $g_{j}(\vec{x})$ and $h_{k}(\vec{x})$, as defined in Eq. (1), to the power of the exponent $\beta$ given in Table 2.

In general, most penalty functions can be classified as static, dynamic, and adaptive penalties [17]. Static penalty means that the penalty parameters are assigned a constant value during the whole optimization process. Dynamic penalty means that the penalty parameters are assigned a value that is changed during the optimization process independent of the optimization variables and constraints. Adaptive penalty means that the penalty parameter is assigned a value that is changed during the optimization process based on the optimization performance. These penalties are listed in Table 2.

\begin{tabular}{|c|c|c|c|}
\hline Penalty & Type & $\beta$ & Penalty Parameter \\
\hline $\mathrm{P} 1$ & Static & 1 & $\lambda$ \\
\hline $\mathrm{P} 2$ & Dynamic & 1 & $\mu * \lambda$ \\
\hline P3 & Dynamic & 2 & $\mu * \lambda$ \\
\hline $\mathrm{P} 4$ & Dynamic & 2 & $\mu+\lambda$ \\
\hline P5 & Dynamic & 1 & $\mu+\lambda$ \\
\hline P6 & Adaptive & 1 & $\begin{array}{l}\left\{\begin{array}{lc}\frac{1}{\mu} \lambda, \quad \tau_{t}>\tau_{\text {target }} \\
\mu \lambda, \quad \text { otherwise }\end{array}\right. \\
\text { numberoffeasibleindividuals }\end{array}$ \\
\hline P7 & Adaptive & 1 & $\begin{array}{c}\tau_{t}=\overline{\text { numberofinfeasibleindividuals }} \\
\qquad f_{\text {avg }}(\vec{x}) * \frac{E_{\text {avg }}(\vec{x})}{\sum E(\vec{x})^{2}}\end{array}$ \\
\hline
\end{tabular}

Table 2. Penalty functions

$\tau_{t}$ is the proportion of feasible individuals, while $\tau_{\text {target }}$ is the targeted proportion of the feasible individuals. In the subsequent sections, the seven penalties will be implemented in MATLAB codes according to the six optimization algorithms (GA, PSO, NM, NM-GA, PSO-GA, NM-PSO) to compare between the effect of using these penalties on the optimization accuracy and robustness.

Two optimization problems are considred for the comparison between the premensioned optimization methods and penalty functions. Our goal is to investigate the effect of using these methods and penalties on the optimization performance. In the first problem, a performance measure is defined to compare between the results of the optimization techniques and penalty methods. In the second problem, a buckling load gain and a mass saving gain are defined to compare between the performance of each optimization method. Details about the two problems and their results are available in the following sections. 


\section{The Minimal Cost Design of a Welded Beam Structure}

The first benchmark problem to be considered in the present investigation is the design optimization of a welded beam structure[18]. As seen in Figure 1, the model consists of a thin cantilevered beam with free length $(L)$ and cross-sectional dimensions $\left(\begin{array}{lll}b & x & t\end{array}\right)$. It is welded over a length $(l)$ to another heavy structural member (rigid support) at its clamped edge. The beam is made of steel 1010 and subjected toa vertical force $P$ at the free end.Such a structural model is used as a benchmark problem to test and compare between the present six optimization techniques.

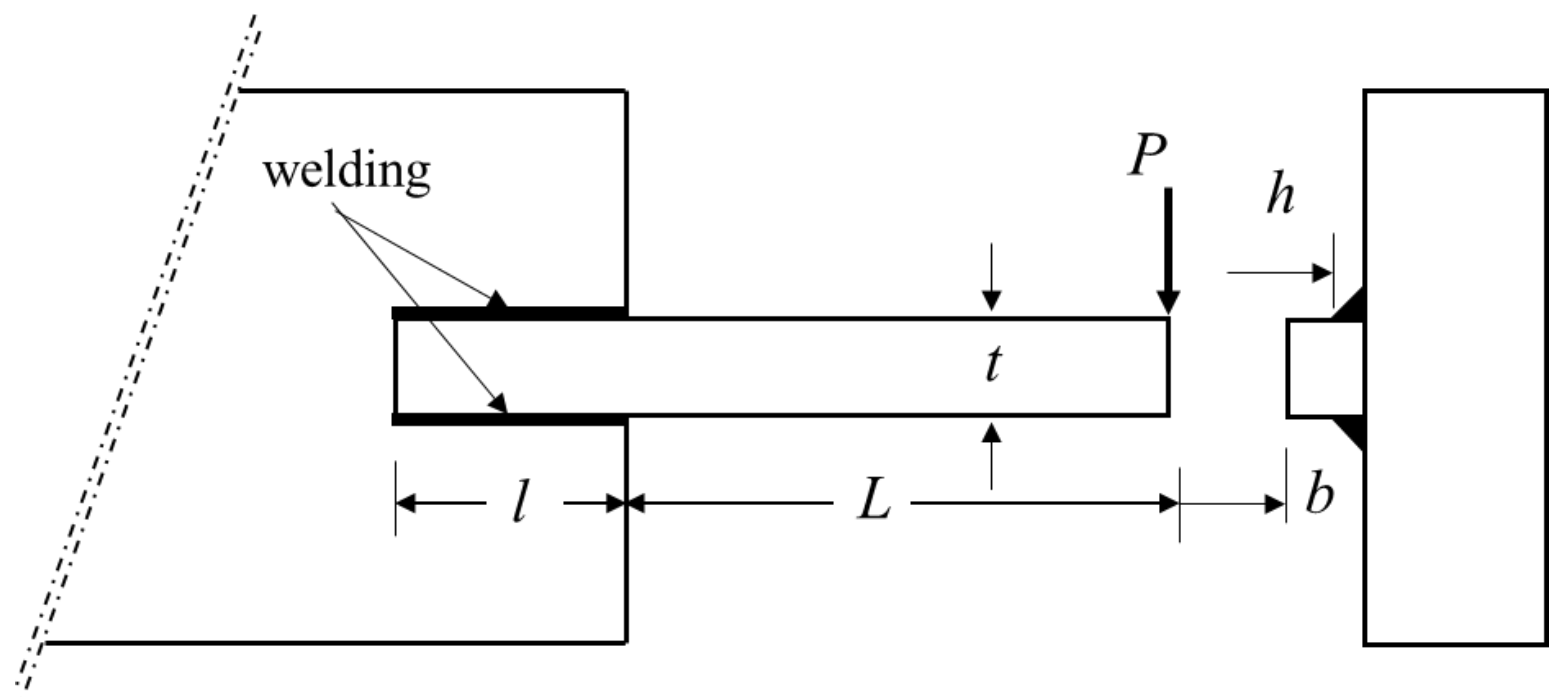

Figure 1. Welded beam design problem

The aim is to find the optimal set of dimensions $\vec{x}=\left(x_{1}, x_{2}, x_{3}, x_{4}\right)=(h, l, t, b)^{T}$ which minimizes the total fabricating cost while satisfying strength requirements as well as the physical bounds imposed on the design variables. According to Sarkar and Roy[18], the associated optimization model is stated in the following:

$$
\begin{array}{cc}
\text { Minimize } & \multicolumn{2}{c}{f(\vec{x})=1.10471 h^{2} l+0.04811 t b(\mathrm{~L}+l)} \\
\text { Subject to the constraints: } & \begin{array}{c}
\tau(\vec{x}) \\
\text { (shear stress) }
\end{array} \\
\text { (bending stress) } & \frac{\sigma(\vec{x})}{\tau_{\text {allow }}}-1 \leq 0 \\
\text { (buckling load) } & 1-\frac{P_{\text {cr }}(\vec{x})}{P} \leq 0 \\
\text { (tip deflection) } & \frac{\delta(\vec{x})}{\delta_{\text {allow }}}-1 \leq 0 \\
\text { (side constraints) } & \frac{h}{b}-1 \leq 0 \\
(h, l, t, b)^{L} \leq(h, l, t, b) \leq(h, l, t, b)^{U}
\end{array}
$$

where $\tau_{\text {allow }}$ denotes the allowable shear stress of weld material, $\sigma_{\text {allow }}$ the allowable stress of the beam material and $\delta_{\text {allow }}$ the allowable value ofbeam tip deflection. Referring to Figure 2, the resultant shear stress in the weld can be calculated from:

$$
\tau(\vec{x})=\sqrt{\tau_{1}{ }^{2}+\tau_{2}{ }^{2}+\frac{2 \tau_{1} \tau_{2}}{\sqrt{1+([h+t] / l)^{2}}}}
$$

where the direct shear stress, $\left(\tau_{1}=\frac{\text { Load }}{\text { throat area }}\right)$, is given by:

$$
\tau_{1}=\frac{P}{\sqrt{2} h l}
$$




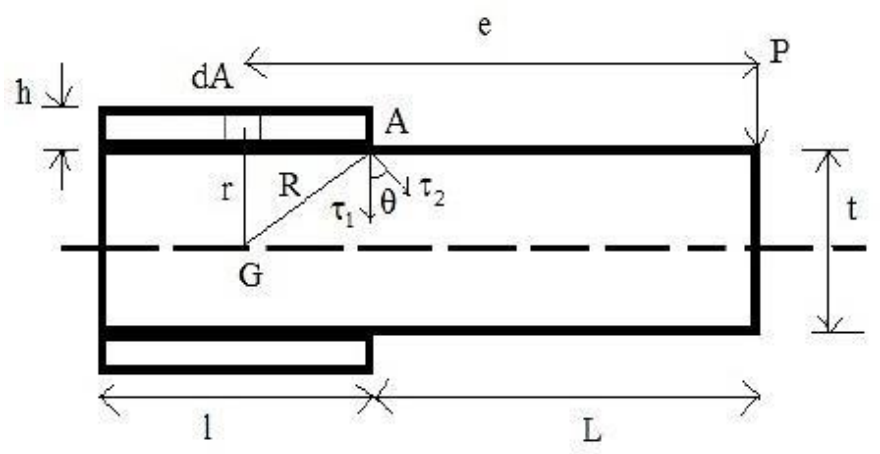

Figure 2. Shear stress components in the weld [18]

The other shear stress component due to the applied turning moment " $P .(L+l / 2)$ " is:

$$
\tau_{2}=\frac{P(L+0.5 l) \sqrt{0.25\left(l^{2}+(h+t)^{2}\right)}}{\sqrt{2} h l\left(\frac{l^{2}}{12}+0.25(h+t)^{2}\right)}
$$

The maximum bending stress and tip deflection of the cantilevered beam are:

$$
\sigma(\vec{x})=\frac{6 P L}{t^{2} b}, \quad \delta(\vec{x})=\frac{4 P L^{2}}{E t^{3} b}
$$

Finally, the critical buckling load, $P_{c r}$, is calculated from the formula [18]:

$$
P_{c r}(\vec{x})=\frac{4.013 \sqrt{E I \alpha}}{L^{2}}\left(1-\frac{t}{2 L} \sqrt{\frac{E I}{\alpha}}\right), I=\frac{t b^{3}}{12}, \alpha=\frac{1}{3} G t b^{3}
$$

where $E$ and $G$ are the Young's and shear moduli of the beam material, respectively.

\section{Optimal Buckling Design of a Functionally Graded Material Column}

The selection of the optimization model is important as well as the optimization algorithm. The seven optimization techniques mentioned before will be implemented for obtaining the optimal buckling design of a functionally graded material (FGM) column under axial compression. This problem has been recently considered by Alshabatat [19], who applied genetic algorithm along with the finite element method to obtain the optimal solutions of a column made of zirconium and aluminium materials. Results were restricted to columns with trigonometric distribution of the volume fractions, which are not easy to manufacture. A practical solutions was given in [20] considering a piecewise structural model of a column constructed from uniform segments, each of which has different volume fraction and length, as shown in Figure 3.

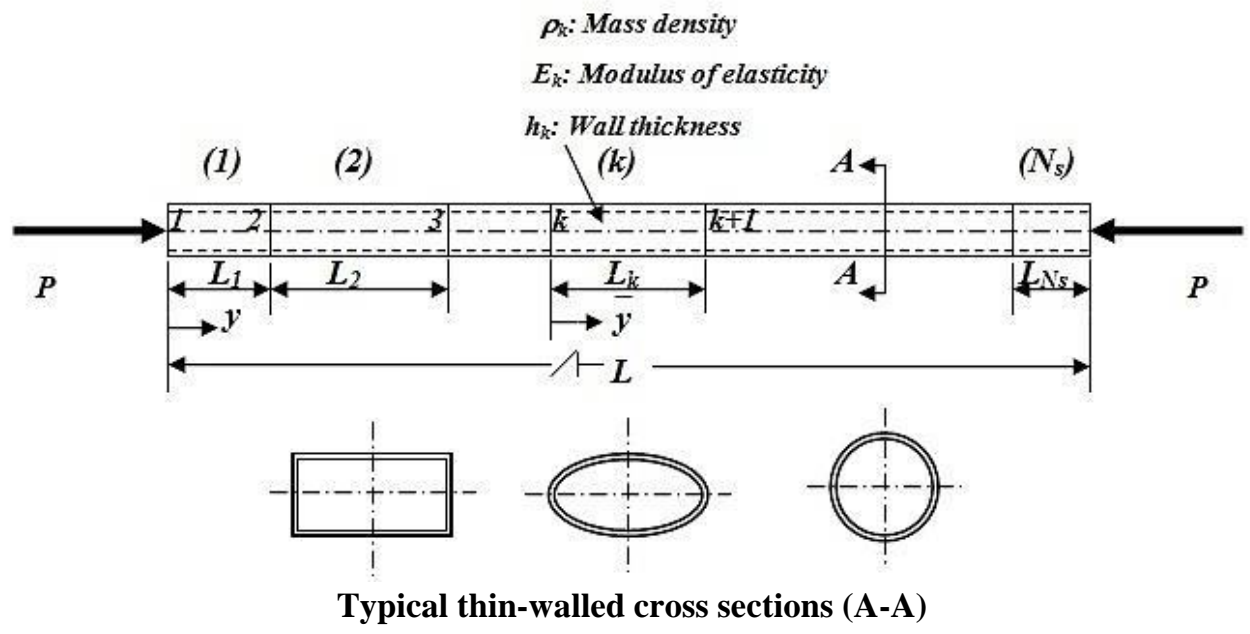

Figure 3. Piecewise structural model of axially compressed slender column [20] 
The governing buckling equation of the $k^{\text {th }}$ segment has the form:

$$
E_{k} I_{k} \frac{d^{4} w}{d y^{4}}+P \frac{d^{2} w}{d y^{2}}=0 ; \quad y_{k} \leq y \leq y_{k+1}
$$

where $E_{k}$ and $I_{k}$ are the Young's Modulus and second moment of area of the $k^{\text {th }}$ segment, respectively. The symbol $w$ denotes the transverse deflection, $P$ is the axial load, and $y$ is the longitudinal coordinate. Eq. (10) can be normalized with respect to a known baseline design that has uniform cross section, stiffness, and mass distributions. Thus, dividing by the known quantity $\left(\frac{E I}{L^{3}}\right)$, one gets:

$$
\frac{d^{4} \widehat{w}}{d \hat{y}^{4}}+\frac{\hat{P}}{\hat{E}_{k} \hat{I}_{k}} \frac{d^{2} \widehat{w}}{d \hat{y}^{2}}=0
$$

where $E, I$, and $L$ are the Young's modulus, second moment of area and column length of the baseline design, respectively. Eq. (11) represents a fourth order differential equation that has the general solution:

$$
\widehat{w}(\hat{y})=a_{1} \sin \left(P_{k} \hat{y}\right)+a_{2} \cos \left(P_{k} \hat{y}\right)+a_{3} \hat{y}+a_{4}
$$

where, $P_{k}=\sqrt{\widehat{\mathrm{P}} / \hat{E}_{k} \hat{I}_{k}}$ and the $a_{i}{ }^{\prime}$ sare constant coefficients that can be obtained by applying the necessary boundary conditions. Definitions of the dimensionless quantities (denoted by the hat symbol) are given in Table 3.

The distributions of the mass density $\rho$ and modulus of elasticity $E$ of any segment $(\mathrm{k})$ are determined from the relations:

$$
\begin{array}{cc}
\text { Mass density : } & \rho_{k}=\rho_{f} V_{f, k+} \rho_{m} V_{m, k} \\
\text { Modulus of elasticity : } & E_{k}=E_{f} V_{f, k+} E_{m} V_{m, k}
\end{array}
$$

where $V_{f, k}$ and $V_{m, k}$ are the fibre and matrix volume fractions of segment $(k)$, respectively. The baseline design is chosen to be constructed from unidirectional composite with the same type of material having equal volume fractions of its constituents, i.e. $V_{f}=V_{m}=50 \%$. It has the same dimensions and wall thickness of the cross-section, i.e. $\hat{I}_{k}=\hat{h}_{k}=1$.

Table 3. Definition of dimensionless quantities

\begin{tabular}{cc}
\hline Quantity & Non-dimensionalization \\
\hline Axial coordinate & $\hat{y}=y / L$ \\
Length of segment (k) & $\hat{L}_{k}=L_{k} / L$ \\
Transverse deflection & $\widehat{w}=w / L$ \\
Wall thickness & $\hat{h}_{k}=h_{k} / h$ \\
Second moment of area & $\hat{I}_{k}=I_{k} / I$ \\
Modulus of elasticity & $\hat{E}_{k}=E_{k} / E$ \\
Bending moment & $\widehat{M}=M *(L / E I)$ \\
Shearing force & $\widehat{F}=F *\left(L^{2} / E I\right)$ \\
Axial force & $\widehat{P}=P *\left(L^{2} / E I\right)$ \\
Mass density & $\hat{\rho}_{k}=\rho_{k} / \rho$ \\
Total structural mass & $\widehat{M}_{s}=\sum_{k=1}^{N s} \hat{\rho}_{k} \hat{h}_{k} \hat{L}_{k}$ \\
\hline
\end{tabular}

For the case of a cantilevered column, the boundary conditions are:

At $\hat{y}=0 \Rightarrow \widehat{w}=0$, and $\hat{\theta}=-\frac{d \widehat{w}}{d \hat{y}}=0$,

At $\hat{y}=1 \Rightarrow \widehat{M}_{s}=-\hat{E}_{k} \hat{I}_{k} \frac{d^{2} \widehat{w}}{d \hat{y}^{2}}=0$, and $\hat{F}=-\hat{E}_{k} \hat{I}_{k} \frac{d^{3} \hat{w}}{d \hat{y}^{3}}+\hat{P} \frac{d \hat{w}}{d \hat{y}}=0$

By applying these boundary conditions and eliminating the $a_{i}{ }^{\prime} s$ coefficients ofEq. (12), the relation between the state variables at both ends of the $k^{\text {th }}$ segment can be obtained in its general matrix form: 


$$
\left\{\begin{array}{c}
\widehat{w}_{k+1} \\
\hat{\theta}_{k+1} \\
\widehat{M}_{k+1} \\
\hat{F}_{k+1}
\end{array}\right\}=\left[\begin{array}{cccc}
1 & -\frac{\operatorname{Sin}\left[\hat{L}_{k} P_{k}\right]}{P_{k}} & \frac{-1+\operatorname{Cos}\left[\widehat{L}_{k} P_{k}\right]}{\hat{P}} & \frac{\operatorname{Sin}\left[\hat{L}_{k} P_{k}\right]-\hat{L}_{k} P_{k}}{\widehat{P} P_{k}} \\
0 & \operatorname{Cos}\left[\hat{L}_{k} P_{k}\right] & \frac{P_{k} \operatorname{Sin}\left[\hat{L}_{k} P_{k}\right]}{\hat{P}} & \frac{1-\operatorname{Cos}\left[\hat{L}_{k} P_{k}\right]}{\hat{P}} \\
0 & -\frac{\widehat{P} \operatorname{Sin}\left[\hat{L}_{k} P_{k}\right]}{P_{k}} & \operatorname{Cos}\left[\hat{L}_{k} P_{k}\right] & \frac{\operatorname{Sin}\left[\hat{L}_{k} P_{k}\right]}{P_{k}} \\
0 & 0 & 0 & 1
\end{array}\right\}\left\{\begin{array}{c}
\widehat{w}_{k} \\
\hat{\theta}_{k} \\
\widehat{M}_{k} \\
\hat{F}_{k}
\end{array}\right\}
$$

Eq. (15) can be extended for any number of segments. For a column constructed from three segments (i.e. $N_{s}=3$ ), the nonlinear buckling equation can be shown to have the form:

$$
P_{2}\left(P_{3}-P_{2} \tan \left[\hat{L}_{2} P_{2}\right] \tan \left[\hat{L}_{3} P_{3}\right]\right)-P_{1} \tan \left[\hat{L}_{1} P_{1}\right]\left(P_{2} \tan \left[\hat{L}_{3} P_{3}\right]+P_{3} \tan \left[\hat{L}_{2} P_{2}\right]\right)=0
$$

Using an appropriate transcendental equation solver, the critical buckling load, $\hat{P}_{c r}$, can be obtained by finding the smallest root ofEq. (16). A MATLAB code is developed to solve this nonlinear equation.

Such an exact buckling analysis can be coupled to a standard nonlinear mathematical programming algorithm for the search of columns designs satisfying the needed design objectives under specified constraints.

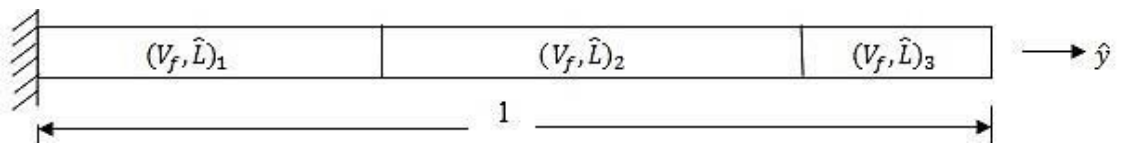

Figure 4. Design variables of a 3-segment cantilevered FGM column

Depending on the form of the objective function, as given in Table 4, the different optimization models to be investigated in the present study are defined in the following:

Find the design variables vector $\vec{x}=\left(V_{f}, \hat{L}\right)_{k=1,2,3}$, which,

$$
\begin{array}{lll} 
& \text { Minimize } & f(\vec{x}) \\
\text { Subject to: } & \text { (Structural mass) } & \widehat{M}_{s}-1.0 \leq 0.0 \\
& \text { (Buckling load) } & 1.0-\left(\frac{4}{\pi^{2}}\right) \widehat{P}_{c r} \leq 0.0 \\
& \text { (Side Constraints) } & \left(V_{f}, \widehat{L}\right)^{L} \leq\left(V_{f}, \widehat{L}\right)_{k=1,2,3} \leq\left(V_{f}, \widehat{L}\right)^{U} \\
& \\
\sum_{k=1}^{N S} \hat{L}_{k}-1.0=0.0
\end{array}
$$

It is worth noting that the constraint $\widehat{M}_{S}-1.0 \leq 0.0$, ensures that the column mass is always less than that of the baseline design. On the other hand, $1.0-\left(\frac{4}{\pi^{2}}\right) \hat{P}_{c r}$ ensures that the critical buckling force of the cantilevered column is greater than that of the baseline design. Notice that $\widehat{M}_{s}$ stands for the ratio of the structural mass to that of the baseline design and $\hat{P}_{c r}$ the dimensionless critical buckling force (refer to Table 3 ).

Table 4. Different forms of the objective function $f(\vec{x})$

\begin{tabular}{lcc}
\hline Optimization Model & Description & Objective function $\boldsymbol{f}(\overrightarrow{\boldsymbol{x}})$ \\
\hline Model-I & Strongest column design & $-\widehat{P}_{c r}$ \\
Model-II & Minimal mass design & $\widehat{M}_{s}$ \\
Model-III & Linear composite objective function & $\widehat{M}_{s}-\left(\frac{4}{\pi^{2}}\right) \widehat{P}_{c r}$ \\
Model-IV & Nonlinear composite objective function & $-\widehat{P}_{c r} / \widehat{M}_{s}$ \\
\hline
\end{tabular}

Side constraints imposed on the fibre volume fraction and lengths are necessary to conform to manufacturing restrictions and avoid having odd-shaped unrealistic column design in the final optimum solutions. In fact, the proper optimization model is found to be important in an optimization process as well as the optimization method. This is totally based on the appropriate selection of objective functions, design variables, and constraints. 


\section{RESULTS AND DISCUSSION}

\section{Case Study (A)}

The seven penalties are implemented in MATLAB codes according to the selected six optimization algorithms. In this case, the welded beam design optimization model, depicted in Figure 1, will be implemented to compare between the effect of using these penalties on the optimization accuracy and robustness. The numerical values of the various preassigned parameters are given in the following[18]:

$$
\begin{aligned}
& P=6000 \mathrm{Ibs}, L=14 \text { inches } \\
& \tau_{\text {allow }}=13600 \mathrm{psi}, \sigma_{\text {allow }}=30000 \mathrm{psi}, \delta_{\text {allow }}=0.25 \text { inches } \\
& E=30 \times 10^{6} \mathrm{psi}, G=12 \times 10^{6} \mathrm{psi} \\
& (0.125,0.1,0.1,0.125) \leq(h, l, t, b) \leq(2,10,10,2) \text { inches }
\end{aligned}
$$

Ten experiments were conducted using the present optimization and penalty methods, on Intel (R), Core (TM), i7$8550 U$ CPU. For each method, the optimum mean value and standard deviation are determined. Then, a performance measure is defined and calculated by multiplying the average error by the average standard deviation for the ten conducted experiments corresponding to each method.The average error is the error sum divided by the number of runs $(n=10)$. The standard deviation measures how widely the values of the equivalent objective function $(F(\vec{x}))$ are far away from the mean value $\left(S T D=\sqrt{\sum\left(F-F_{\text {mean }}\right)^{2} /(n-1)}\right)$. Thus, the error measures the accuracy of the optimization algorithm, and the standard deviation determines its robustness. For this reason, the multiplication of both the error and standard deviation can be used to measure the method general performance. This performance measure is used to weigh the robustness and accuracy of each penalty function as well as each optimization algorithm. As the performance measure goes lower and lower the method becomes more robust and stable. The performance measures for both the optimization algorithms and penalty functions are illustrated in Figures 5 and 6, respectively.

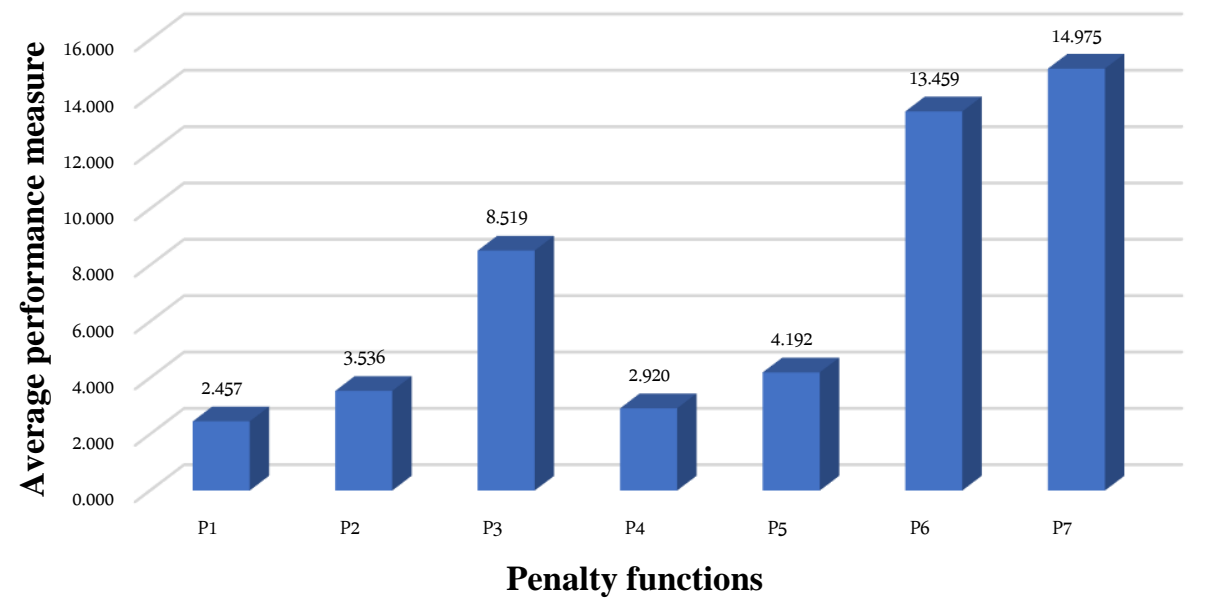

Figure 5. Average penalties performance measure

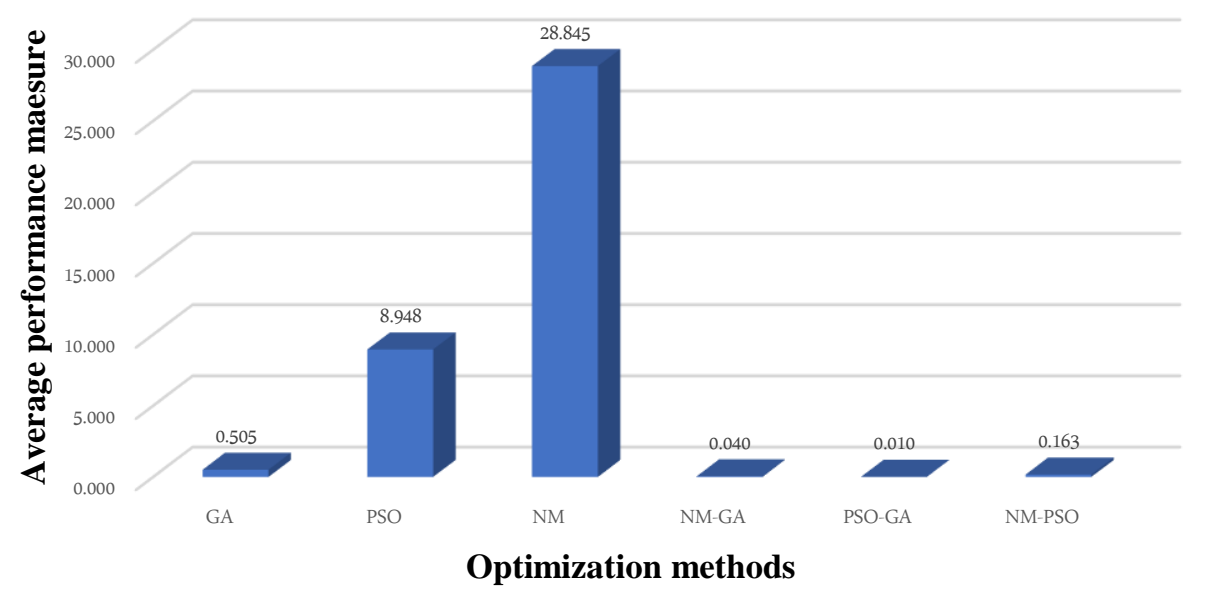

Figure 6. Average optimization methods performance measure 
It is worth noting thatthe minimum value of the cost function is obtained by NM-GA with the penalty function P4 that is $f_{\min }=2.3813$ with design variables $\vec{x}_{o p t}=(h, l, t, b)=(0.2436,6.1398,8.4045,0.2436)$.

Table 5 lists the best, mean, and worst values of the objective corresponding to each optimization algorithm and penalty function. In case of GA, the best optimum value is obtained for adaptive penalty P7, while the worst values are obtained for the dynamic penalty P2 and the adaptive penalty P6, in point of view of error. For the PSO, the best values are obtained using the dynamic penalty $\mathrm{P} 3$, while the worst values are obtained using the adaptive penalty P7. In case of the NM, the best values are obtained using the dynamic penalty $\mathrm{P} 2$, while the worst values are obtained using the adaptive penalty P6. The adaptive penalty P7 does not work well with the NM because the objective function violates the applied constraints. In other words, the adaptive penalty P7 does not compatible with the Nelder-Meade method in its present form. In case of GA-NM and GA-PSO, the results are approximately near each other for all the penalty functions. This can be attributes to the power of the hybrid models in which most of the results are near the optimum value. But the results in case of the GA-PSO are in general better than the other two hybrid models, for the present optimization problem. However, the best optimum solution is obtained by the PSO-GA, based on the performance measure.

The beam welded problem is solved using the MATLAB SQP algorithm, where the obtained minimum was 2.3811 for the cost function with the corresponding values of design variable $\vec{x}=(0.2444,6.2186,8.2915,0.2444)$ that is little bit smaller than the value obtained by NM-GA.

Table 5. Best mean, and worst values of the objective function for each optimization algorithm and penalty function for the welded beam

\begin{tabular}{cccccccccc}
\hline \multirow{2}{*}{ Penalty function } & \multicolumn{3}{c}{ GA } & \multicolumn{3}{c}{ PSO } & \multicolumn{3}{c}{ NM } \\
\cline { 2 - 10 } & Fbest & Fmean & Fworst & Fbest & Fmean & Fworst & Fbest & Fmean & Fworst \\
\hline P1 & 3.7644 & 4.54624 & 5.8471 & 5.0918 & 6.90622 & 9.2857 & 2.5354 & 8.65332 & 16.2714 \\
P2 & 4.5652 & 4.94317 & 5.2233 & 4.6453 & 6.97498 & 8.5058 & 2.4894 & 8.44347 & 24.8258 \\
P3 & 4.0816 & 4.88711 & 5.7213 & 3.0148 & 5.00947 & 7.9364 & 3.0473 & 12.01171 & 26.4884 \\
P4 & 4.1067 & 4.67988 & 5.9823 & 3.5437 & 6.14399 & 8.5587 & 2.6716 & 8.81796 & 22.0698 \\
P5 & 3.7275 & 4.5472 & 5.4629 & 3.7037 & 5.81596 & 6.5979 & 2.5024 & 7.54406 & 16.0636 \\
P6 & 3.9989 & 5.11727 & 6.6283 & 4.1302 & 7.08116 & 9.8176 & 6.3559 & 17.04125 & 27.7266 \\
P7 & 3.4952 & 4.3238 & 5.2371 & 6.9768 & 9.65051 & 11.8991 & - & - & - \\
\hline
\end{tabular}

\begin{tabular}{ccccccccccc}
\hline \multirow{2}{*}{ Penalty function } & \multicolumn{3}{c}{ NM-GA } & \multicolumn{3}{c}{ PSO- GA } & \multicolumn{3}{c}{ NM-PSO } \\
\cline { 2 - 10 } & Fbest & Fmean & Fworst & Fbest & Fmean & Fworst & Fbest & Fmean & Fworst \\
\hline P1 & 2.41 & 2.53967 & 2.8675 & 2.3834 & 2.41277 & 2.4909 & 2.4516 & 3.24378 & 4.4198 \\
P2 & 2.3816 & 2.59838 & 3.9491 & 2.3845 & 2.50145 & 2.6533 & 2.4716 & 2.98982 & 3.7047 \\
P3 & 2.3817 & 2.62236 & 4.0212 & 2.3943 & 2.62348 & 3.352 & 2.4837 & 2.9824 & 3.8693 \\
P4 & 2.3813 & 2.73492 & 4.4904 & 2.3977 & 2.44256 & 2.5585 & 2.5543 & 3.174 & 4.1993 \\
P5 & 2.4166 & 2.51803 & 2.717 & 2.4149 & 2.51929 & 2.7344 & 2.6705 & 3.3793 & 4.3839 \\
P6 & 2.3863 & 2.65843 & 3.5624 & 2.4306 & 2.60932 & 3.2403 & 2.6567 & 3.07984 & 4.1057 \\
P7 & 2.3861 & 2.70568 & 3.478 & 2.3956 & 2.47751 & 2.6534 & 2.7227 & 3.32988 & 4.6325 \\
\hline
\end{tabular}

Table 6. The function error and standard deviation for each algorithm and penalty function for the welded beam

\begin{tabular}{ccccccc}
\hline \multirow{2}{*}{ Penalty } & \multicolumn{2}{c}{ GA } & \multicolumn{3}{c}{ PSO } & \multicolumn{2}{c}{ NM } \\
\cline { 2 - 7 } & STD & Error & STD & Error & STD & Error \\
\hline P1 & 0.561 & 0.909 & 1.267 & 1.9 & 4.4076 & 2.634 \\
P2 & 0.176 & 1.0759 & 1.171 & 1.929 & 7.313 & 2.546 \\
P3 & 0.523 & 1.0524 & 1.636 & 1.104 & 12.0117 & 4.044 \\
P4 & 0.531 & 0.965 & 1.59 & 1.58 & 6.566 & 2.168 \\
P5 & 0.537 & 0.9096 & 7.0811 & 1.442 & 6.566 & 2.168 \\
P6 & 0.693 & 1.149 & 7.0812 & 1.974 & 10.685 & 6.158 \\
P7 & 0.599 & 0.816 & 9.651 & 3.0529 & - & - \\
\hline
\end{tabular}




\begin{tabular}{ccccccc}
\hline \multirow{2}{*}{ Penalty } & \multicolumn{2}{c}{ NM-GA } & \multicolumn{2}{c}{ PSO-GA } & \multicolumn{2}{c}{ NM-PSO } \\
\cline { 2 - 7 } & STD & Error & STD & Error & STD & Error \\
\hline P1 & 0.1457 & 0.0666 & 0.0343 & 0.0133 & 0.559 & 0.362 \\
P2 & 0.4519 & 0.0912 & 0.0941 & 0.051 & 0.398 & 0.256 \\
P3 & 0.472 & 0.1013 & 0.3 & 0.102 & 0.388 & 0.252 \\
P4 & 0.603 & 0.1486 & 0.0478 & 0.0258 & 0.507 & 0.333 \\
P5 & 0.105 & 0.0575 & 0.1 & 0.058 & 0.4786 & 0.419 \\
P6 & 0.347 & 0.116 & 0.228 & 0.0958 & 0.452 & 0.293 \\
P7 & 0.354 & 0.1363 & 0.093 & 0.04 & 0.5865 & 0.398 \\
\hline
\end{tabular}

\section{Case Study B}

To investigate the role of the model formulation in the whole optimization process, four different optimization strategies were implemented, as given in Table 4, to find the required optimal solutions of a cantilevered $F G M$ column depicted in Figure 4. Two types of materials are considered: Carbon/Epoxy and E-glass/Epoxy with their properties listed in Table 7.

Table 7. Material properties of selected fiber-reinforced composites [20]

\begin{tabular}{ccccc}
\hline \multirow{2}{*}{ Material type } & \multicolumn{2}{c}{ Fibers } & \multicolumn{2}{c}{ Matrix } \\
\cline { 2 - 5 } & $\boldsymbol{\rho}_{\boldsymbol{f}}\left[\boldsymbol{g} / \mathbf{c m}^{\mathbf{3}}\right]$ & $\boldsymbol{E}_{\boldsymbol{f}}[\mathbf{G P a}]$ & $\boldsymbol{\rho}_{\boldsymbol{m}}\left[\mathbf{g} / \mathbf{c m}^{\mathbf{3}}\right]$ & $\boldsymbol{E}_{\boldsymbol{m}}[\mathbf{G P a}]$ \\
\hline Carbon/Epoxy & 1.81 & 235.0 & 1.27 & 4.3 \\
E-glass/Epoxy & 2.54 & 73.0 & 1.27 & 4.3 \\
\hline
\end{tabular}

Firstly, the resulting solutions were compared by implementing the six optimization techniques including the $N M$, $G A, S Q P, N M-G A, P S O-G A$, and NM-PSO. All these optimization algorithms are applied to the strongest column design model(Model-I) defined in Table 4. A three-segment cantilevered column is considered first, which is made of Carbon/Epoxy composite assuming the lower and upper limiting values of the fiber volume fraction to be $10 \%$ and $90 \%$, respectively.The attained optimal solutions are listed in Table 8 , wherethe corresponding optimization gain represents the percentage increase in the buckling load as compared with that of the baseline design value $\left(\widehat{P}_{c r}=\frac{\pi^{2}}{4}\right)$. It is seen that the best solutions are attained by utilizing either the $S Q P$ or the hybrid $P S O-G A$, where the optimization gain reaches its maximum value of $17.95 \%$ with the total structural mass maintained constant. Comes next, the $N M-P S O$ with $16.9 \%$ gain and then the $N M-G A$ with $16.52 \%$. As a general observation, the hybrid algorithms have proved to be much efficient and robust than the stand-alone ones,based on the performance measure of the present problem.

Considering next, solutions obtained by applying the $S Q P$ method for global optimization [21] to the different models defined in Table 4 . The attained results are presented in Table 9, considering manufacturing constraints as expressed by assigning values of $25 \%$ and $75 \%$ to the lower and upper limits imposed on the fiber volume fraction. It is seen that, the optimization gain obtained by applying Model-I decreased by about $1.34 \%$ than that obtained without manufacturing constraints as indicated in Table 8.Although, Models III and $I V$ represent two different multi-objective optimization models (linear and nonlinear combination of the mass and buckling load), they obtained the same results according to the present optimization problem. Implementation of Model-IIresults in a littlemass saving of $2.4 \%$ while preserving the critical buckling load at its baseline value of 2.4675 . This was expected because the wall thickness is not taken as a design variable in the present formulation.It is to be noticed that the $S Q P$ can only deal with optimization models described by continuous design variables, which represents an obstacle when one is considering discrete variables for some practical engineering applications. 
Table 8. Optimization results based on Model-I for different optimization algorithms $\left(\widehat{M}_{s}=1\right)$ (Case of a cantilevered FGM column made of carbon/epoxy composites)

\begin{tabular}{ccc} 
& $(0.1,0.2) \leq\left(V_{f}, \widehat{L}\right)_{k=1,2,3} \leq(0.9,0.8)$ & \\
\hline $\begin{array}{c}\text { Optimization } \\
\text { Algorithm }\end{array}$ & $\begin{array}{c}\text { Optimal Design Vector } \\
\overrightarrow{\boldsymbol{x}}_{\boldsymbol{o p t}}=\left(\boldsymbol{V}_{\boldsymbol{f}}, \widehat{\boldsymbol{L}}\right)_{\boldsymbol{k}=\mathbf{1}, 2,3}\end{array}$ & $\begin{array}{c}\text { Buckling Load } \\
\widehat{\boldsymbol{P}}_{\boldsymbol{c r} \text {, max }}(\text { Gain \% })^{*}\end{array}$ \\
\hline $\mathrm{SQP}$ & $(0.6945,0.5348)_{1},(0.3992,0.2652)_{2},(0.1136,0.2)_{3}$ & $2.9104(17.95 \%)$ \\
$\mathrm{NM}$ & $(0.4789,0.409)_{1},(0.3953,0.3341)_{2},(0.6697,0.2569)_{3}$ & $2.8185(14.23 \%)$ \\
GA & $(0.7434,0.2905)_{1},(0.536,0.4598)_{2},(0.1505,0.2497)_{3}$ & $2.8726(16.42 \%)$ \\
NM-GA & $(0.667,0.4905)_{1},(0.4572,0.2635)_{2},(0.2129,0.246)_{3}$ & $2.875(16.52 \%)$ \\
PSO-GA & $(0.6945,0.5348)_{1},(0.3992,0.2652)_{2},(0.1136,0.2)_{3}$ & $2.9104(17.95 \%)$ \\
NM-PSO & $(0.6885,0.4161)_{1},(0.5159,0.3228)_{2},(0.1801,0.2611)_{3}$ & $2.8842(16.9 \%)$ \\
\hline *Gain \% $=\left(\left(\frac{4}{\pi^{2}}\right) \hat{P}_{c r, \text { max }}-1.0\right) * 100$ &
\end{tabular}

Table 9. Optimal solutions based on SQP for the different optimization models (Case of a cantilevered FGM column made of carbon/epoxy composites)

$$
(0.25,0.2) \leq\left(V_{f}, \widehat{L}\right)_{k=1,2,3} \leq(0.75,0.8)
$$

\begin{tabular}{cccc}
\hline $\begin{array}{c}\text { Optimization } \\
\text { Model }\end{array}$ & $\begin{array}{c}\text { Optimal Design Vector } \\
\overrightarrow{\boldsymbol{x}}_{\boldsymbol{o p t}}=\left(\boldsymbol{V}_{\boldsymbol{f}}, \widehat{\boldsymbol{L}}\right)_{\boldsymbol{k}=1,2,3}\end{array}$ & $\begin{array}{c}\text { Buckling Load } \\
\widehat{\boldsymbol{P}}_{\boldsymbol{c}}(\mathbf{G a i n} \%)\end{array}$ & $\begin{array}{c}\text { Structural Mass } \\
\widehat{\boldsymbol{M}}_{\boldsymbol{s}}(\text { Saving \%)* }\end{array}$ \\
\hline Model-I & $(0.6721,0.4724) 1,(0.4590,0.2415) 2,(0.25,0.2858) 3$ & $2.8722(16.61 \%)$ & $1.0(0.0 \%)$ \\
Model-II & $(0.575,0.4434) 1,(0.413,0.2296) 2,(0.25,0.327) 3$ & $2.4674(0.0 \%)$ & $0.976(2.4 \%)$ \\
Model-III & $(0.6721,0.4724) 1,(0.4590,0.2415) 2,(0.25,0.2858) 3$ & $2.8722(16.61 \%)$ & $1.0(0.0 \%)$ \\
Model-IV & $(0.6721,0.4724) 1,(0.4590,0.2415) 2,(0.25,0.2858) 3$ & $2.8722(16.61 \%)$ & $1.0(0.0 \%)$ \\
\hline
\end{tabular}

$*$ Mass Saving $\%=\left(1.0-\widehat{M}_{s, \min }\right) * 100$

For more deep understanding of what happens inside the design spaces, the effect of changing some variables on the maximum critical buckling load under mass constraint was investigated. Columndesigns near the calculated optimal solution, obtained by implementing Model-I, are presented in Figures 7 and 8, showing the developed level curves of the critical buckling load in the design spaces $\left(V_{f}, \widehat{L}\right)_{1}$ and $\left(V_{f 2}, V_{f 3}\right)$, respectively.

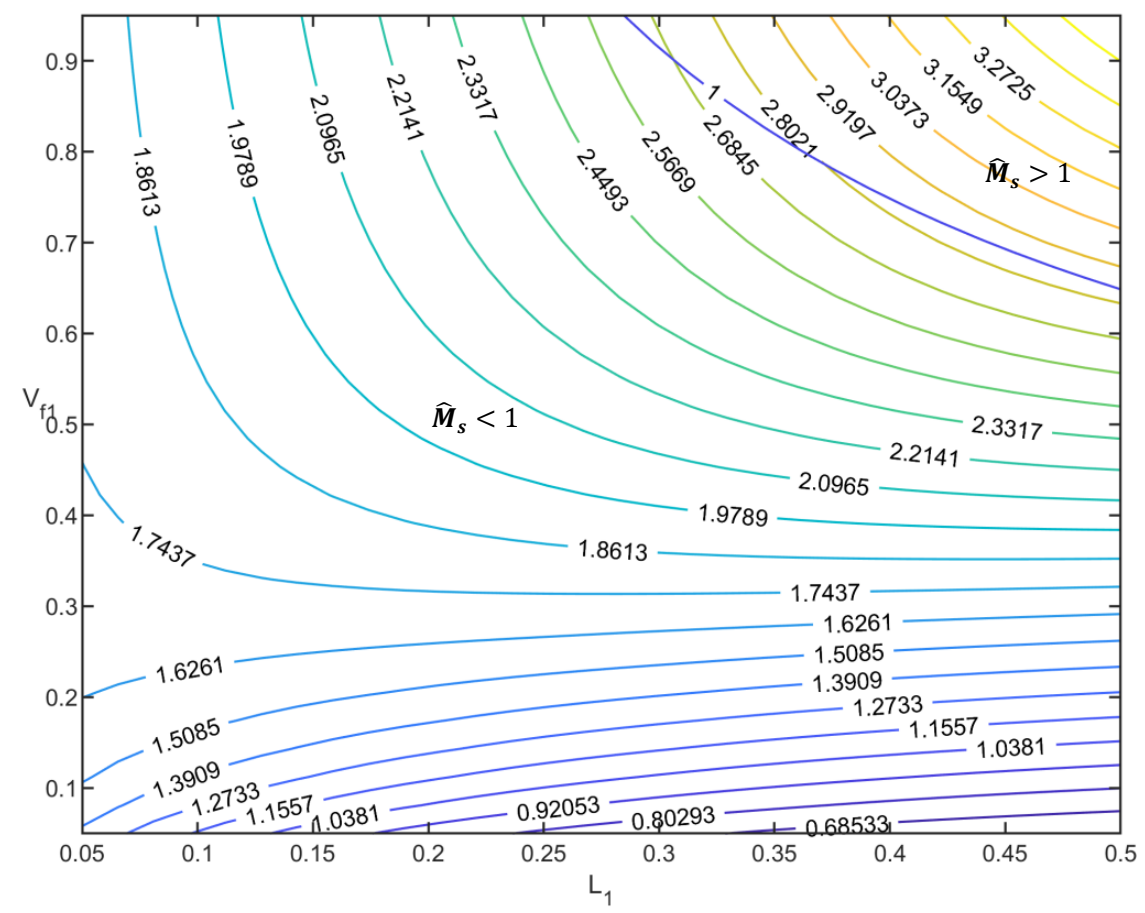

Figure 7. Level curves of $\widehat{P}_{c r}$ in the design space $\left(V_{f}, \widehat{L}\right)_{1}$ near the optimal solution attained by Model-I 


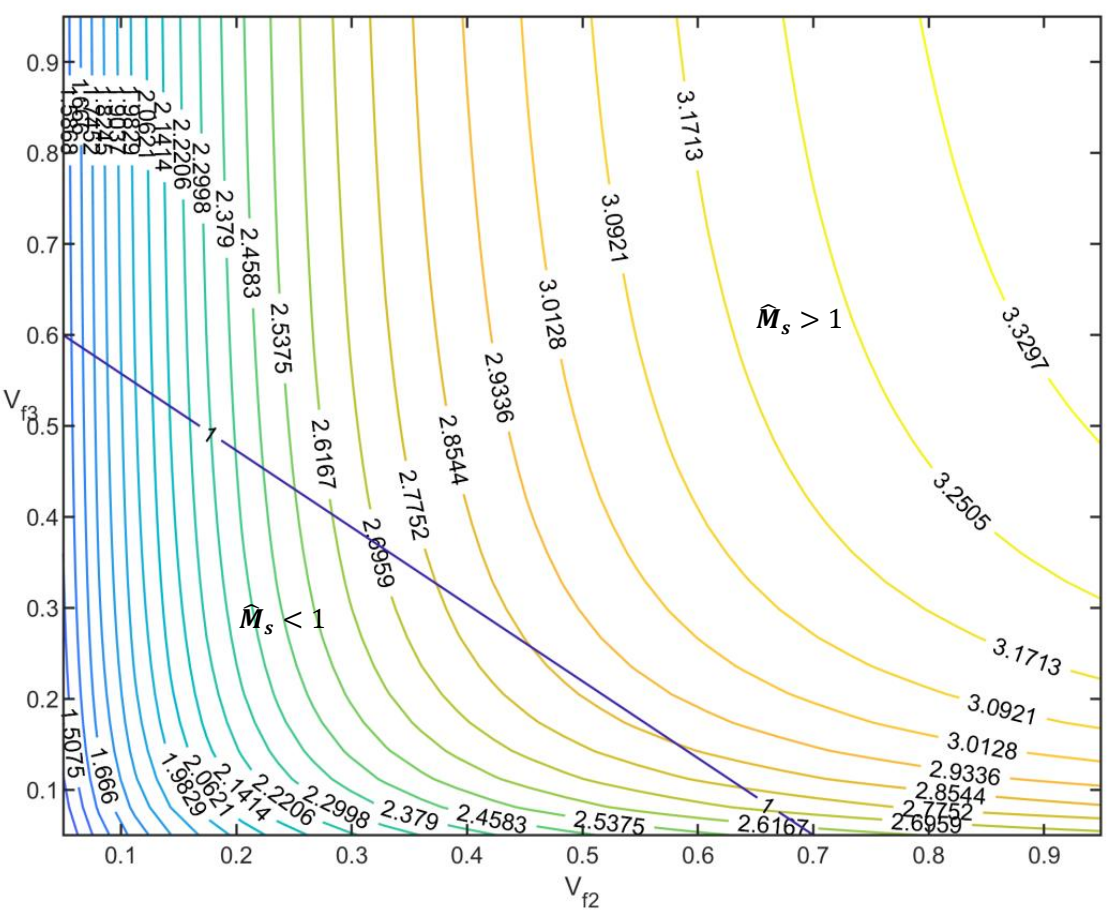

Figure 8. Level curves of $\widehat{P}_{c r}$ in the design space $\left(V_{f 2}, V_{f 3}\right)$ near the optimal solution attained by Model-I

It is seen that the buckling load, even though implicit function in the design variables, is well behaved, monotonic and defined everywhere in the selected design spaces. The blue line, which represents the locus of constant mass $\widehat{M}_{s}=1$, divides the space into two domains: the lower domain for structural mass less than that of the baseline design (i.e. $\widehat{M}_{s}<$ 1 ), and the upper for $\widehat{M}_{s}>1$. As shown in Figure 7, the critical buckling load increases with increasing $V_{f 1}$, however there is no significant change with increasing $\hat{L}_{1}$. According to Figure 8, both $V_{f 2}$ and $V_{f 3}$ nearly have the same effect on $\hat{P}_{c r}$, however the effect of $V_{f 2}$ is more significant.

More results have been obtained to see the effect of changing the material type with different physical and mechanical properties. The results for E-glass/epoxy are listed in Table 10, where it can be observed that Model-II results in a balanced improvement in both mass and buckling load.

Table 10 . Optimal solutions based on SQP for the different optimization models (Case of a cantilevered FGM column made of E-glass/Epoxy composites)

\begin{tabular}{cccc}
\hline $\begin{array}{c}\text { Optimization } \\
\text { Model }\end{array}$ & $\begin{array}{c}\text { Optimal Design Vector } \\
\overrightarrow{\boldsymbol{x}}_{\boldsymbol{p} \boldsymbol{t} \boldsymbol{t}}=\left(\boldsymbol{V}_{\boldsymbol{f}}, \widehat{\boldsymbol{L}}\right)_{\boldsymbol{k}=\mathbf{1 , 2 , 3}}\end{array}$ & $\begin{array}{c}\text { Buckling Load } \\
\widehat{\boldsymbol{P}}_{\boldsymbol{c} \boldsymbol{r}}(\text { Gain \%) }\end{array}$ & $\begin{array}{c}\text { Structural Mass } \\
\widehat{\boldsymbol{M}}_{\boldsymbol{s}}(\text { Saving \%) }\end{array}$ \\
\hline Model-I & $(0.6836,0.459) 1,(0.4661,0.2359) 2,(0.25,0.3051) 3$ & $2.8592(15.88 \%)$ & $1.0(0.0 \%)$ \\
Model-II & $(0.575,0.4434) 1,(0.413,0.2296) 2,(0.25,0.327) 3$ & $2.4813(0.56 \%)$ & $0.9544(4.56 \%)$ \\
Model-III & $(0.6836,0.459) 1,(0.4661,0.2359) 2,(0.25,0.3051) 3$ & $2.8592(15.88 \%)$ & $1.0(0.0 \%)$ \\
Model-IV & $(0.6836,0.459) 1,(0.4661,0.2359) 2,(0.25,0.3051) 3$ & $2.8592(15.88 \%)$ & $1.0(0.0 \%)$ \\
\hline
\end{tabular}

\section{CONCLUSIONS}

This paper investigates different optimization algorithms and models that can be applied and implemented to optimize nonlinear programming design problems. Seven robust algorithms and seven penalty functions are implemented in MATLAB codes and experimented using carefully selected benchmark problems. For a specific design problem, the selection of the proper optimization algorithm along with the proper penalty function is found to be crucial in the whole optimization process. The penalty functions are classified as static, dynamic or adaptive penalties, which have been thoroughly examined for handling design constraints. Implementations of these techniques to practical optimization models in mechanical and structural design have revealed that the performance of the static and dynamic penalties outweighs the performance of the adaptive penalties. The best result has been achieved using the static penalty in reaching the needed optimal solutions. More results have indicated that the hybrid algorithm $(N M-G A)$ with the dynamic penalty function $P 4$ is superior in performance when compared with the stand-alone, single ones. This has been confirmed by 
implementing the different algorithms for solving the welded beam structural model where the objective function is measured by minimizing the total fabricating cost of the welded structure subject to various nonlinear stress, buckling and deflection as well as side constraints.

In addition of implementing a robust optimization algorithm, the model formulation is also so crucial in reaching the required global optimal solutions. Four different optimization strategies were compared to determine the effect of changing the objective function and constraints on the attained optimal design of a FGM column under axial compression. The volume fractions of the two constituents' material are allowed to vary piece-wisely, making the physical and mechanical properties to be tailored in the axial direction. Numerical solutions and detailed results for a 3-segment cantilevered column, which are based on dimensionless analysis, are given and useful design charts have been developed and analysed. It is proved that the buckling load, even though it is an implicit function in the design variables, is well behaved, monotonic and defined everywhere in the selected design space. In all, the best solutions have been reached by applying the hybrid PSO-GA and PSO-NM and the SQP method based on global search. However, the major disadvantageous of the latter is that it deals only with models described by continuous design variables.

Future studies will consider more implementation of new robust hybrid algorithms for solving similar mechanical and structural design models, such as the stability and dynamic optimization of sandwich constructions with either material or thickness grading along predetermined directions.

\section{REFERENCES}

[1] A. Ravindran, G. V. Reklaitis, and K. M. Ragsdell, Engineering optimization: methods and applications, 2nd ed. Hoboken, N.J: John Wiley \& Sons, 2006.

[2] G. Venter, "Review of optimization techniques," Encyclopedia of aerospace engineering, 2010 [Online]. Available: http://onlinelibrary.wiley.com/doi/10.1002/9780470686652.eae495/full. [Accessed: 16-Dec-2015]

[3] O. Yeniay, "A comparative study on optimization methods for the constrained nonlinear programming problems," Mathematical Problems in Engineering, vol. 2005, no. 2, pp. 165-173, 2005, doi: 10.1155/MPE.2005.165.

[4] A. Al-Garni and A. H. Kassem, "On the optimization of aerospace plane ascent trajectory," AIAA/CIRA 13th International Space Planes and Hypersonics Systems and Technologies Conference, p. 3293, 2007.

[5] R. J. Kuo and C. C. Huang, "Application of particle swarm optimization algorithm for solving bi-level linear programming problem," Computers \& Mathematics with Applications, vol. 58, no. 4, pp. 678-685, Aug. 2009, doi: 10.1016/j.camwa.2009.02.028.

[6] S.-K. S. Fan and E. Zahara, "A hybrid simplex search and particle swarm optimization for unconstrained optimization," European Journal of Operational Research, vol. 181, no. 2, pp. 527-548, Sep. 2007, doi: 10.1016/j.ejor.2006.06.034.

[7] Z.-W. Ren, "Hybrid simplex-improved genetic algorithm for global numerical optimization," ACTA AUTOMATICA SINICA, vol. 33, no. 1, p. 0091, 2007, doi: 10.1360/aas-007-0091.

[8] E. Zahara and Y.-T. Kao, "Hybrid Nelder-Mead simplex search and particle swarm optimization for constrained engineering design problems," Expert Systems with Applications, vol. 36, no. 2, pp. 3880-3886, Mar. 2009, doi: 10.1016/j.eswa.2008.02.039.

[9] R. C. Eberhart, J. Kennedy, and others, “A new optimizer using particle swarm theory," in Proceedings of the sixth international symposium on micro machine and human science, 1995, vol. 1, pp. 39-43.

[10] Q. Bai, “Analysis of particle swarm optimization algorithm," Computer and Information Science, vol. 3, no. 1, p. $180,2010$.

[11] H. Holland, Adaptation in Natural and Artificial Systems. University of Michigan Press, 1975.

[12] C. Guo and X. Yang, "A programming of genetic algorithm in Matlab 7.0,” Modern Applied Science, vol. 5, no. 1, 2011.

[13] R. B. Wilson, “A simplicial algorithm for concave programming,” Harvard, 1963.

[14] R. Fletcher, "The sequential quadratic programming method," in Nonlinear Optimization, Springer, 2010, pp. $165-214$.

[15] H. Rahami, A. Kaveh, M. Aslani, and R. N. Asl, "A hybrid modified genetic-nelder mead simplex algorithm for large-scale truss optimization," International Journal of Optimization in Civil Engineering, vol. 1, no. 1, pp. 29-46, 2011.

[16] P. Nanakorn and K. Meesomklin, "An adaptive penalty function in genetic algorithms for structural design optimization," Computers \& Structures, vol. 79, no. 29-30, pp. 2527-2539, Nov. 2001, doi: 10.1016/S0045-7949(01)00137-7.

[17] S. B. Hamida and M. Schoenauer, "An Adaptive Algorithm for Constrained Optimization Problems," in Parallel Problem Solving from Nature PPSN VI, 2000, pp. 529-538, doi: 10.1007/3-540-45356-3_52.

[18] M. Sarkar and T. K. Roy, "Multi-objective welded beam optimization using Neutrosophic goal programming technique," Advances in Fuzzy Mathematics, vol. 12, no. 3, pp. 515-538, 2017.

[19] N. T. Alshabatat, "Optimal design of functionally graded material columns for buckling problems," Journal of Mechanical and Engineering Sciences, vol. 12, no. 3, pp. 3914-3926, Sep. 2018, doi: 10.15282/jmes.12.3.2018.11.0342.

[20] K. Y. Maalawi, “Optimization of elastic columns using axial grading concept," Engineering Structures, vol. 31, no. 12, pp. 2922-2929, Dec. 2009, doi: 10.1016/j.engstruct.2009.07.017.

[21] "MATLAB - MathWorks." [Online]. Available: https://www.mathworks.com/products/matlab.html 